Pacific Journal of Mathematics

THE HYPERBOLIC METRIC AND COVERINGS OF RIEMANN 


\title{
THE HYPERBOLIC METRIC AND COVERINGS OF RIEMANN SURFACES
}

\section{Carl David Minda}

\begin{abstract}
Suppose $X$ and $Y$ are Riemann surfaces which have the open unit ball as universal covering surface. Let $d \sigma_{X}, d \sigma_{Y}$ be the hyperbolic metric on $X, Y$, respectively. Given any analytic function $f: X \rightarrow Y$ the principle of hyperbolic metric asserts that $\left(f^{*}\left(d \sigma_{Y}\right) / d \sigma_{X}\right)(p) \leqq 1$ for each point $p \in X$ where $f^{*}\left(d \sigma_{Y}\right)$ is the pull-back to $X$ via $f$ of the hyperbolic metric on $Y$. Moreover, equality holds if and only if $f$ is an (unbranched, unlimited) covering of $X$ onto $Y$. This paper has two main objectives. The first is to study how the principle of hyperbolic metric can be strengthened if we only consider analytic functions which are not coverings. The second is to investigate the set of all analytic coverings of $X$ onto $Y$.
\end{abstract}

1. Notation and terminology. Throughout this paper, unless the contrary is explicitly stated, $X$ and $Y$ will always denote Riemann surfaces whose universal covering surface is the open unit ball $\boldsymbol{B}$. The set of all analytic functions $f: X \rightarrow Y$ will be denoted by $A(X, Y)$. Often we will fix points $p \in X, q \in Y$ and consider analytic functions $f:(X, p) \rightarrow(Y, q)$. This notion implies that $f(p)=q$.

We shall make free use of the theory of covering surfaces. For example, the material in [3, pp. 27-44] or [15, Ch. 5] is sufficient for our purposes. To say that $f: X \rightarrow Y$ is an analytic covering projection will always mean that $X$ is an (unbranched, unlimited) covering surface of $Y$ and $f$ is an analytic function. Let $C(X, Y)$ denote the set of all analytic coverings $f: X \rightarrow Y$; it is possible that $C(X, Y)$ is empty. $N(X, Y)$ is the complement of $C(X, Y)$ in $A(X, Y)$. One basic result we shall need is the following. Suppose $f: X \rightarrow Y$, $g: Y \rightarrow Z$, and $h: X \rightarrow Z$ are analytic mappings of Riemann surfaces such that $g \circ f=h$. Then if any two of these functions are coverings, so is the third. In particular, the composition of coverings is again a covering.

Given a Riemann surface $X$ with the unit ball as universal covering surface, there is a unique conformal metric $d \sigma_{X}=\lambda_{X}(z)|d z|$ of constant curvature -4 on $X$ called the hyperbolic metric. It is defined on $X$ by projecting the Poincaré hyperbolic metric on $\boldsymbol{B}$ onto $X$ by means of any analytic universal covering projection. For any analytic function $f: X \rightarrow Y$ we will let $f^{*}\left(d \sigma_{Y}\right)$ denote the pull-back to $X$ via the function $f$ of the hyperbolic metric on $Y$. Note that if $\pi: B \rightarrow X$ is an analytic universal covering projection, then $\pi^{*}\left(d \sigma_{X}\right)=d \sigma_{B}$. If $d s_{1}=\rho_{1}(z)|d z|$ and $d s_{2}=\rho_{2}(z)|d z|$ are two conformal 
metrics on $X$, then the quotient $d s_{1} / d s_{2}$ defines a function on $X$.

The fundamental group of $X$ with base point $p$ will be denoted by $\pi_{1}(X, p)$. As is well-known each analytic function $f:(X, p) \rightarrow(Y, q)$ induces a group homomorphism $f_{*}: \pi_{1}(X, p) \rightarrow \pi_{1}(Y, q)$ defined by $f_{*}([\gamma])=[f \circ \gamma]$, where $[\gamma]([f \circ \gamma])$ denotes the homotopy class of the closed path $\gamma(f \circ \gamma)$ based at $p(q)$.

2. The principle of hyperbolic metric. Suppose $f: X \rightarrow Y$ is an analytic function. The principle of hyperbolic metric asserts that

$$
\frac{f^{*}\left(d \sigma_{Y}\right)}{d \sigma_{X}}(p) \leqq 1
$$

for each point $p \in X$. We want to determine when equality holds at a point. In order to do this it is necessary to recall the brief proof of the principle of hyperbolic metric. Fix $p \in X$ and set $q=f(p)$. Let $\pi:(B, 0) \rightarrow(X, p)$ and $\omega:(B, 0) \rightarrow(Y, q)$ be analytic universal covering projections. Then there is a unique analytic function $\widetilde{f}:(\boldsymbol{B}, 0) \rightarrow(\boldsymbol{B}, 0)$ such that $f \circ \pi=\omega \circ \widetilde{f}$. The inequality (1) is equivalent to $\left|\tilde{f}^{\prime}(0)\right| \leqq 1$ and equality holds in (1) if and only if it holds in this inequality. Thus, equality holds if and only if $\widetilde{f}$ is a rotation of $\boldsymbol{B}$; that is, if and only if $\widetilde{f}:(\boldsymbol{B}, 0) \rightarrow(\boldsymbol{B}, 0)$ is an analytic covering. From $f \circ \pi=\omega \circ \tilde{f}$ we see that $\tilde{f}$ is a covering if and only if $f$ is a covering. Therefore, in the principle of hyperbolic metric we get equality at a point if and only if $f$ is a covering. Of course, if $f$ is a covering, then equality holds at every point of $X$.

One question that we shall investigate is whether the inequality (1) can be improved if we restrict our attention to the class $N(X, Y)$ of analytic functions which are not coverings. To be more precise we define a function $\Omega: X \times Y \rightarrow[0,1]$ by $\Omega(p, q)=\sup \left\{f^{*}\left(d \sigma_{Y}\right) / d \sigma_{X}(p)\right.$ : $f \in N(X, Y), f(p)=q\}$. Our program is two-fold. First, we want to investigate the behavior of the function $\Omega$. Unless $X$ is simply connected we shall see that $\Omega$ is pointwise strictly less than one. We will also consider the possibility of uniform estimates of $\Omega$. Secondly, we shall study the class $C(X, Y)$ of all analytic coverings of $X$ onto $Y$. Generally, $C(X, Y)$ is not a very large subset of $A(X, Y)$.

For the special case $X=Y$ and $p=q$ the function $\Omega(p, p)$ was first studied by Aumann and Carathéodory [4]. For an annulus the actual value of $\Omega(p, p)$ was rather implicitly determined by Heins [6] and Hervé [9]. A more explicit and more elementary determination of this constant and the extremal functions for an annulus was given by Minda [16].

3. An improved version of the principle of hyperbolic metric. 
We begin by establishing an analog of Schwarz' lemma.

Proposition 1. Suppose $a \in \boldsymbol{B} \backslash\{0\}$ and $0 \leqq \delta<1$. Let $\mathscr{\mathscr { S }}(a, \delta)$ be the set of all analytic functions $f:(\boldsymbol{B}, 0) \rightarrow(\boldsymbol{B}, 0)$ which also satisfy $|f(a)| \leqq \delta|a|$. Then

$$
\mu=\max \left\{\left|\dot{f}^{\prime}(0)\right|: f \in \mathscr{B}(a, \delta)\right\}=\frac{|a|+\delta}{1+|a| \delta} .
$$

Also, $f \in \mathscr{B}(a, \delta)$ is extremal if and only if $f(z)=e^{i \phi} h\left(e^{i 0} z\right)$, where $0=-\operatorname{Arg} a$ and $h(z)=z(\mu-z) /(1-\mu z)$.

Proof. First, note that we may assume $a \in(0,1)$ and $f(a) \in[0,1)$. If this were not true, then simply consider $e^{-i \phi} f\left(e^{-i \theta} z\right)$, where $\theta=-$ $\operatorname{Arg} a$ and $\phi=\operatorname{Arg} f(a)$. Now, $f \in \mathscr{B}(\alpha, \delta)$ satisfies this normalization if and only if there is an analytic function $g: \boldsymbol{B} \rightarrow \boldsymbol{B}$ with $f(z)=$ $z g(z)$ and $0 \leqq g(a) \leqq \delta$. In this case $g(0)=f^{\prime}(0)$. Let $\mathscr{B} \mathscr{B}_{0}$ be the set of all such functions $g$, then $\mu=\max \left\{|g(0)|: g \in \mathscr{B}_{0}\right\}$. Now, it is elementary to determine $\mu$. Since $0 \leqq g(\alpha) \leqq \delta$, the point $g(0)$ must lie in the closed hyperbolic ball with center $g(a)$ and radius $1 / 2 \log$ $(1+a) /(1-a)$, which is the hyperbolic distance from 0 to $a$. This hyperbolic ball is symmetric about the real axis and intersects the real axis in the two points $[g(a)-a] /[1-a g(a)]$ and $[g(\alpha)+a] /$ $[1+a g(a)]$. The latter point is farther from the origin so

$$
|g(0)| \leqq \frac{a+g(a)}{1+a g(a)} \leqq \frac{a+\delta}{1+a \delta}=\mu .
$$

Equality holds if and only if $g(z)=(\mu-z) /(1-\mu z)$. This establishes the proposition.

THEOREM 1. Let $X$ and $Y$ be Riemann surfaces and assume that $X$ is not simply connected. Then the function $\Omega$ is pointwise strictly less than one.

Proof. Fix analytic universal covering projections $\pi:(\boldsymbol{B}, 0) \rightarrow$ $(X, p)$ and $\omega:(B, 0) \rightarrow(Y, q)$. Because $X$ is not simply connected, there is a point $\widetilde{p} \in \boldsymbol{B} \backslash\{0\}$ with $\pi(\widetilde{p})=p$. We may assume that $|\widetilde{p}|$ is minimal among all such points. Now, $\omega^{-1}(\{q\})$ is a discrete subset of $\boldsymbol{B}$ so there are just finitely many points in $\omega^{-1}(\{q\})$ with modulus strictly less than $|\widetilde{p}|$. Hence, there is a constant $\delta, 0 \leqq \delta<1$, such that if $\widetilde{q} \in \omega^{-1}(\{q\})$ and $|\widetilde{q}|<|\widetilde{p}|$, then actually $|\widetilde{q}| \leqq \delta|\widetilde{p}|$.

Consider any $f \in N(X, Y)$ with $f(p)=q$. There is a unique analytic function $\tilde{f}:(\boldsymbol{B}, 0) \rightarrow(\boldsymbol{B}, 0)$ such that $f \circ \pi=\omega \circ \widetilde{f}$. From $\omega(\widetilde{f}(\widetilde{p}))=f(\pi(\widetilde{p}))=f(p)$ we see that $\widetilde{f}(\widetilde{p}) \in \omega^{-1}(\{q\})$. Because $f$ is not 
a covering, the function $\widetilde{f}$ is not a rotation of $\boldsymbol{B}$. Hence, $|\widetilde{f}(\widetilde{p})|<|\widetilde{p}|$ by Schwarz' lemma. The preceding paragraph implies that $|\widetilde{f}(\widetilde{p})| \leqq \delta|\widetilde{p}|$, where $\delta$ is independent of the particular function $f$. By Proposition 1 we have

$$
\left|\tilde{f}^{\prime}(0)\right| \leqq \frac{|\widetilde{p}|+\delta}{1+|| \widetilde{p} \mid \delta}=\mu<1
$$

Hence,

$$
\frac{f^{*}\left(d \sigma_{Y}\right)}{d \sigma_{X}}(p) \leqq \mu,
$$

which gives $\Omega(p, q) \leqq \mu$. This establishes the theorem.

In the special case $X=Y$ and $p=q$ this result is due to Aumann and Carathéodory [4]. For this reason the number $\Omega(p, q)$ will be called the Aumann-Carathéodory rigidity constant. Actually, to exactly recover the theorem of Aumann and Carathéodory it is necessary to observe that every covering $f:(X, p) \rightarrow(X, p)$ must be a conformal automorphism of $X$.

4. The modular spectrum of a Riemann surface. In this section we recall some basic facts about the modular spectrum of a Riemann surface which will be employed in later sections. For details the reader is referred to the work of Huber [10], Marden, Richards and Rodin [14] and Jenkins and Suita [11].

Let $X$ be a Riemann surface. Given a closed curve $\gamma$ on $X$ let $\{\gamma\}$ be the class of all closed curves on $X$ which are freely homotopic to $\gamma$. The module $M_{X}(\gamma)$ of $\{\gamma\}$ is the greatest lower bound of the set of all hyperbolic lengths of the curves in $\{\gamma\}$. The modular spectrum of $X$ is the set of all nonnegative real numbers $M_{X}(\gamma)$, where $\gamma$ ranges over all closed curves on $X$ which are not freely homotopic to a point. A number $M$ in the modular spectrum is said to have finite multiplicity if there are only finitely many distinct free homotopy classes $\{\gamma\}$ with $M_{X}(\gamma)=M . X$ is said to have a discrete modular spectrum if the modular spectrum is a discrete subset of the real numbers. Finally, $X$ has a finite modular spect$r u m$ if the modular spectrum is discrete and every nonzero element of the spectrum has finite multiplicity.

There is an alternate way to express the modulus of a free homotopy class which uses covering surfaces. Let $\{\gamma\}$ be a nontrivial free homotopy class on $X$. Then $\{\gamma\}$ uniquely determines a covering surface $\tilde{X}(\gamma)$ of $X$ whose fundamental group is infinite cyclic [14]. In fact, $\widetilde{X}(\gamma)$ is conformally equivalent to the punctured ball $\boldsymbol{B} \backslash\{0\}$ or to a proper annulus $A_{R}=\{z: 1 / R<|z|<R\}$ for 
some $R>1$ according as $M_{X}(\gamma)$ is zero or positive. If $M_{X}(\gamma)>0$, then we can take $\widetilde{X}(\gamma)=A_{R}$, where $R$ is uniquely determined by $M_{X}(\gamma)=\pi^{2} / 2 \log R$. Also, if $M_{X}(\gamma)>0$, then there is a unique curve $\gamma_{0}$ in $\{\gamma\}$ whose hyperbolic length is equal to $M_{X}(\gamma)$. The curve $\gamma_{0}$ is the image of the unit circle if we represent $\widetilde{X}(\gamma)$ as the annulus $A_{R}$ [11]. When $\gamma$ is not freely homotopic to a point, $M_{X}(\gamma)=0$ if and only if $\gamma$ is retractable to an isolated pointlike boundary element [14]. Such a closed curve is called a point-cycle. An isolated pointlike boundary element is a point of the ideal boundary which is isolated and has a neighborhood in $X$ conformally equivalent to a punctured disk. $A$ surface $X$ possesses a closed curve $\gamma$ with $M_{X}(\gamma)>0$ except when $X$ is conformally equivalent to $B$ or $B \backslash\{0\}$.

Proposition 2. Suppose $X$ and $Y$ are Riemann surfaces, $\gamma$ is a closed curve on $X$ and $f \in A(X, Y)$. Then $M_{Y}(f \circ \gamma) \leqq M_{X}(\gamma)$. If $M_{X}(\gamma)>0$, then equality holds if and only if $f$ is a covering.

Proof. The inequality is well-known ([10], [11], [14]); it is a simple consequence of the principle of hyperbolic metric. Our interest is in determining when equality holds. Since a covering preserves the hyperbolic metric and $\{f \circ \gamma\}=\{f \circ \delta: \delta \in\{\gamma\}\}$, it is clear that equality holds if $f$ is a covering. On the other hand, suppose equality holds and $M_{X}(\gamma)>0$. We may assume that $\gamma$ is the unique curve in $\{\gamma\}$ with minimal hyperbolic length. Then

$$
M_{Y}(f \circ \gamma) \leqq \int_{f \circ \gamma} d \sigma_{Y}=\int_{\gamma} f^{*}\left(d \sigma_{Y}\right) \leqq \int_{\gamma} d \sigma_{X}=M_{X}(\gamma) .
$$

Since equality must hold throughout, we see that $f^{*}\left(d \sigma_{Y}\right) / d \sigma_{X}=1$ on $\gamma$ so by the principle of hyperbolic metric $f$ must be a covering.

Observe that the proof shows that if $f: X \rightarrow Y$ is a covering and $\gamma_{0}$ is the unique curve in $\{\gamma\}$ with hyperbolic length equal to $M_{X}(\gamma)$, then $f \circ \gamma_{0}$ is the unique curve in $\{f \circ \gamma\}$ with hyperbolic length equal to $M_{Y}(f \circ \gamma)$.

5. Coverings of Riemann surfaces. We now investigate the set $C(X, Y)$ of all coverings of the Riemann surface $X$ onto the Riemann surface $Y$. We shall see that there are many analogies between this set and the group $\mathscr{A}(X)$ of conformal automorphisms of a Riemann surface. Since $\mathscr{A}(X) \subset C(X, X)$, many known results concerning the group $\mathscr{A}(X)$ follow as corollaries of our work. We shall not explicitly cite these corollaries.

THEOREM 2. Let $X$ and $Y$ be Riemann surfaces and assume $X$ has nonabelian fundamental group. Given any sequence $\left(f_{n}\right)_{n=1}^{\infty}$ of 
distinct functions in $C(X, Y)$ and any point $p \in X$, the sequence $\left(f_{n}(p)\right)_{n=1}^{\infty}$ has no limit point in $Y$.

Proof. Suppose there were a sequence $\left(f_{n}\right)_{n=1}^{\infty}$ of distinct functions in $C(X, Y)$ and a point $p \in X$ with $f_{n}(p) \rightarrow q \in Y$. By a result of Komatu and Mori [12] we may even assume that $\left(f_{n}\right)_{n=1}^{\infty}$ converges to an analytic function $f: X \rightarrow Y$ uniformly on every compact subset of $X$. The uniformity of convergence is with respect to the hyperbolic distance. Let $\pi:(B, 0) \rightarrow(X, p)$ and $\omega:(B, 0) \rightarrow(Y, q)$ be analy tic universal covering projections. Take an open ball $B$ centered at the origin such that $\omega \mid B$ is injective. We assume that $f_{n}(p) \in \omega(B)$ for all $n$. Let $\widetilde{f}_{n}: \boldsymbol{B} \rightarrow \boldsymbol{B}$ be the unique analytic function which satisfies $\widetilde{f}_{n}(0) \in B$ and $f_{n} \circ \pi=\omega \circ \widetilde{f}_{n}$. Each $\widetilde{f}_{n}$ is a conformal automorphism of $\boldsymbol{B}$ since it is a self-covering of $\boldsymbol{B}$. Then $\left(\tilde{f}_{n}\right)_{n=1}^{\infty}$ converges uniformly on compact subsets of $\boldsymbol{B}$ to a conformal automorphism $\widetilde{f}:(\boldsymbol{B}, 0) \rightarrow$ $(\boldsymbol{B}, 0)$ which satisfies $f \circ \pi=\omega \circ \widetilde{f}$.

Let $\Gamma(\Delta)$ be the group of cover transformations associated with the covering $\pi(\omega)$. Since $\widetilde{f}_{n} \Gamma \subset \Delta \widetilde{f}_{n}, \widetilde{f} \Gamma \subset \Delta \widetilde{f}$, the functions $\widetilde{f}_{n}, \widetilde{f}$ induce group homomorphisms $f_{n}^{*}, f^{*}: \Gamma \rightarrow \Delta$ defined by $f_{n}^{*}(T)=$ $\widetilde{f}_{n} \circ T \circ \widetilde{f}_{n}^{-1}, f^{*}(T)=\widetilde{f} \circ T \circ \widetilde{f}^{-1}$. Consequently, $f_{n}^{*}(T)=\left(\widetilde{f}_{n} \circ \widetilde{f}^{-1}\right) \circ f^{*}(T) \circ$ $\left(\widetilde{f} \circ \widetilde{f}_{n}^{-1}\right)$. Now, $\widetilde{f}_{n} \circ \widetilde{f}^{-1}$ converges to the identity function uniformly on compact subsets of $B$, so it follows that $f_{n}^{*}(T) \rightarrow f^{*}(T)$ uniformly on compact subsets of $\boldsymbol{B}$ for each $\boldsymbol{T} \in \boldsymbol{B}$. But the group $\Delta$ is discrete, so there is an integer $n(T)$ such that $f_{n}^{*}(T)=f^{*}(T)$ for all $n \geqq n(T)$. This implies that $\left(\widetilde{f}^{-1} \circ \widetilde{f}_{n}\right) \circ T=T \circ\left(\widetilde{f}^{-1} \circ \widetilde{f}_{n}\right)$, or the Möbius transformations $\tilde{f}^{-1} \circ \widetilde{f}_{n}$ and $T$ commute for $n \geqq n(T)$. Since $\tilde{f}^{-1} \circ \widetilde{f}_{n}$ is not the identity, we may conclude for $T$ not the identity that $\widetilde{f}^{-1} \circ \widetilde{f}_{n}$ and $T$ have the same fixed points on $\partial B$ for $n \geqq n(T)[13$, p. 72]. A simple argument shows that all nontrivial $T$ in $\Gamma$ have the same fixed points which implies that the group $\Gamma$ is abelian. Since $\Gamma$ is isomorphic to the fundamental group of $X$, this establishes the theorem.

Simple examples show that this theorem is false for any surface $X$ with abelian fundamental group. For a Riemann surface $X$ with nonabelian fundamental group Theorem 2 shows that the set $C(X, Y)$ is countable and for fixed $p \in X, q \in Y$ the set of all coverings $f:(X, p) \rightarrow(Y, q)$ is finite.

THEOREM 3. Let $X$ and $Y$ be Riemann surfaces and suppose $X$ has nonabelian fundamental group. Given $f \in C(X, Y)$ there does not exist a sequence $\left(f_{n}\right)_{n=1}^{\infty}$ in $A(X, Y)$ such that $f_{n} \rightarrow f$ uniformly on compact subsets of $X$ and $f_{n} \neq f$ for all $n$.

Proof. Fix a closed curve $\gamma$ on $X$ with $M_{X}(\gamma)>0$. Suppose 
that such a sequence $\left(f_{n}\right)_{n=1}^{\infty}$ did exist. Without loss of generality we may assume that all the $f_{n}$ are distinct. Then $f_{n} \circ \gamma$ is freely homotopic to $\mathbf{f} \circ \gamma$ for all $n$ sufficiently large. Therefore, $M_{Y}\left(f_{n} \circ \gamma\right)=$ $M_{Y}(f \circ \gamma)$ for all $n$ large enough. Now, $M_{Y}(f \circ \gamma)=M_{X}(\gamma)$ since $f$ is a covering. Then $M_{Y}\left(f_{n} \circ \gamma\right)=M_{X}(\gamma)$ which implies that $f_{n}$ must also be a covering for all $n$ sufficiently large. We now assume $f_{n}$ is a covering for all $n$. Fix $p \in X$. Then $f_{n}(p) \rightarrow f(p) \in Y$ which violates Theorem 2. This contradiction proves Theorem 3.

Theorem 3 simultaneously generalizes a theorem of Heins [7] and Theorem 1. Note that Theorem 3 is false for any surface with an abelian fundamental group. However, the following holds for any surface $X$ conformally equivalent to an annulus: There is no sequence in $N(X, Y)$ which converges to an element of $C(X, Y)$. The same argument as in the proof of Theorem 3 establishes this result which will be used later.

THEOREM 4. Suppose X has nonabelian fundamental group and $Y$ has finite modular spectrum. Then the set $C(X, Y)$ is finite.

Proof. Take any closed curve $\gamma$ on $X$ with $M_{X}(\gamma)>0$. Without loss of generality we may assume that $\gamma$ is the unique closed curve in its free homotopy class with minimal hyperbolic length. For any $f \in C(X, Y)$ we have $M_{Y}(f \circ \gamma)=M_{X}(\gamma)$. Since $Y$ has finite modular spectrum there are just finitely many distinct free homotopy classes $\left\{\delta_{1}\right\}, \cdots,\left\{\delta_{n}\right\}$ on $Y$ with $M_{Y}\left(\delta_{i}\right)=M_{X}(\gamma)$. We suppose that each $\delta_{i}$ has minimal hyperbolic length in its free homotopy class. Then each $f \in C(X, Y)$ maps $\gamma$ onto some $\delta_{i}$. Let $C_{i}(1 \leqq i \leqq n)$ be the set of all $f \in C(X, Y)$ which map $\gamma$ onto $\delta_{i}$; it is enough to show that each $C_{i}$ is a finite set. Fix a point $p$ on the curve $\gamma$. For each $f \in C_{i}$ the point $f(p)$ belongs to the compact set $\delta_{i}$. Now, Theorem 2 implies that the set $C_{i}$ is finite.

The question of whether a Riemann surface with nonabelian fundamental group can have a self-covering which is not a conformal automorphism was first raised by Heinz Hopf [8]. Such a covering is called nontrivial. Note that the punctured disk, once punctured plane and a torus have abelian fundamental group and possess nontrivial coverings. On the other hand, the annulus theorem [14] implies that every self-covering of an annulus is trivial. The work of Huber [10] implies that every Riemann surface with finite modular spectrum and nonabelian fundamental group has no nontrivial covering. Heins [8] showed that if a Riemann surface has no Green's function, then it also has no nontrivial coverings. The next theorem generalizes Huber's result about the existence of nontrivial coverings. 
THEOREM 5. Let $X$ be a Riemann surface with nonabelian fundamental group. If some number in the modular spectrum of $X$ has finite multiplicity, then every self-covering of $X$ is a conformal automorphism.

Froof. First, we consider the case in which some positive number $M$ in the modular spectrum has finite multiplicity. Suppose $\left\{\gamma_{1}\right\}, \cdots,\left\{\gamma_{n}\right\}$ are the only distinct free homotopy classes with $M_{X}\left(\gamma_{i}\right)=M$. If $f: X \rightarrow X$ is any covering, then $M_{X}\left(f \circ \gamma_{i}\right)=M$ so $f \circ \gamma_{1}$ is freely homotopic to $\gamma_{i}$ for some unique integer $i, 1 \leqq i \leqq n$. Let $f^{(n)}$ denote the $n$ 'th iterate of $f$. Then $f^{(n)} \circ \gamma_{1}$ is freely homotopic to $\gamma_{i(n)}$. This implies that there exist distinct integers $1 \leqq j<k$ with $f^{(j)} \circ \gamma_{1}$ freely homotopic to $f^{(k)} \circ \gamma_{1}$, or $f^{(k-j)}$ maps $f^{(j)} \circ \gamma_{1}$ onto a curve which is freely homotopic to itself. Since $M_{X}\left(f^{(j)} \circ \gamma_{1}\right)>0$, this implies that $f^{(k-j)}$ is a conformal automorphism of $X$ [14]. Therefore, $f$ itself is a conformal automorphism.

Now, suppose zero belongs to the spectrum of $X$ and has finite multiplicity. Then $X$ has finitely many isolated pointlike boundary elements. This means that there is a Riemann surface $Y \supset X$ such that $Y \backslash X$ is a finite nonempty set of points and $Y$ has no isolated pointlike boundary elements. By a generalized version of Picard's theorem [14] $f$ extends to an analytic self-map of $Y$. Since $f$ is a self-cover of $X$, its extension to $Y$ must map $Y \backslash X$ into itself. As in the preceding case there is an integer $j$ such that $f^{(j)}$ fixes one point $p \in Y \backslash X$. Then either $f$ is a conformal automorphism of $Y$ or else $f^{(n j)}$ converges to $p$ uniformly on compact subsets of $Y$ [14]. This would imply that for any closed curve $\gamma$ on $X, M_{X}\left(f^{(n j)} \circ \gamma\right)=0$ for all $n$ sufficiently large. This is a contradiction because each iterate of $f$ is again a covering.

Similar arguments show that the hypotheses of Theorem 5 imply that the group of conformal automorphisms of $X$ is finite.

6. Bounds for the Aumann-Carathéodory rigidity constant. In this section we shall be interested in obtaining upper and lower bounds for $\Omega$. We are especially interested in obtaining bounds which are independent of either the first or the second variable.

We begin with an observation about the existence of extremal functions. Let $A(X, Y)$ be given the topology of uniform convergence on compact subsets. Note that Theorem 3 and the comment immediately after its proof imply that $N(X, Y)$ is a closed subset of $A(X, Y)$ if $X$ is not conformally equivalent to $\boldsymbol{B}$ or $\boldsymbol{B} \backslash\{0\}$. In particular, for such a surface $X$ and fixed points $p \in X, q \in Y$ the set of all $f \in N(X, Y)$ with $f(p)=q$ is compact. This means that if $X$ is not conformally equivalent to $B$ or $B \backslash\{0\}$, then there exists 
a function $f \in N(X, Y)$ with $\Omega(p, q)=\left(f^{*}\left(d \sigma_{Y}\right) / d \sigma_{X}\right)(p)$, where $f(p)=q$.

Proposition 3. Suppose $X$ is a Riemann surface which is not conformally equivalent to $\boldsymbol{B}$ or $\boldsymbol{B} \backslash\{0\}$. Then for any Riemann surface $Y$ the function $\Omega$ is upper semicontinuous in each variable.

Proof. Fix $p \in X$ and let $\left(p_{n}\right)_{n=1}^{\infty}$ be any sequence in $X$ which converges to $p$. For each $n$ let $f_{n}$ be an extremal function for $\Omega\left(p_{n}, q\right)$. From the work of Komatu and Mori [12] it follows that there is a subsequence $\left(f_{n_{j}}\right)_{j=1}^{\infty}$ which converges to $f \in A(X, Y), f(p)=q$, uniformly on compact subsets of $X$. From the preceding remarks we know that $f \in N(X, Y)$. Clearly,

$$
\frac{f_{n_{j}}^{*}\left(d \sigma_{Y}\right)}{d \sigma_{X}}\left(p_{n_{j}}\right) \longrightarrow \frac{f^{*}\left(d \sigma_{Y}\right)}{d \sigma_{X}}(p),
$$

so that $\Omega\left(p_{n_{j}}, q\right) \rightarrow \Omega(p, q)$. This implies that $\lim \sup _{r \rightarrow p} \Omega(r, q) \leqq \Omega(p, q)$, so that $\Omega$ is upper semicontinuous in the first variable. A similar argument demonstrates upper semicontinuity in the second variable.

Suppose $X$ is not conformally equivalent to $B$ or $B \backslash\{0\}$. If $K$ is a compact subset of $X$ and $L$ a compact subset of $Y$, then $\Omega$ is uniformly less than one on $K \times L$ since an upper semicontinuous function assumes its maximum value on a compact set. In particular, this is true if $X$ and $Y$ are compact. If just $Y$ is compact, then for a fixed $p \in X \Omega(p, q)$ is bounded by a constant less than one, independent of $q \in Y$. This result will be generalized to certain noncompact surfaces in Theorem 7 .

Now, we obtain a lower bound for $\Omega$. Let $T(X, Y)$ be the set of all analytic mappings $f: X \rightarrow Y$ such that the induced homomorphism $f_{*}: \pi_{1}(X, p) \rightarrow \pi_{1}(Y, f(p))$ is trivial. Define $\widetilde{\Omega}: X \times Y \rightarrow[0,1]$ by

$$
\widetilde{\Omega}(p, q)=\sup \left\{\frac{f^{*}\left(d \sigma_{Y}\right)}{d \sigma_{X}}(p): f \in T(X, Y), f(p)=q\right\} .
$$

Clearly, $\widetilde{\Omega}(p, q) \leqq \Omega(p, q)$. It is elementary to express $\widetilde{\Omega}$ as the quotient of two conformally invariant metrics.

THEOREM 6. $\widetilde{\Omega}(p, q)=c_{B}(p) / \lambda_{X}(p)$, where $c_{B}(z)|d z|$ is the analytic capacity metric on $X$ and $\lambda_{X}(z)|d z|$ is the hyperbolic metric on $X$. In particular, $\widetilde{\Omega}(p, q)$ does not depend on $q$.

Proof. Let $\omega:(B, 0) \rightarrow(Y, q)$ be an analytic universal covering projection. Since $f_{*}$ is trivial, each function $f \in T(X, Y)$ lifts to a unique analytic function $\tilde{f}:(X, p) \rightarrow(\boldsymbol{B}, 0)$ such that $\pi \circ \widetilde{f}=f$. 
Conversely, any analytic function $\widetilde{f}:(X, p) \rightarrow(\boldsymbol{B}, 0)$ defines a function $f$ in $T(X, Y)$ by $f=\pi \circ \widetilde{f}$. Since

$$
f^{*}\left(d \sigma_{Y}\right)=\widetilde{f}^{*}\left(\pi^{*}\left(d \sigma_{Y}\right)\right)=\widetilde{f}^{*}\left(d \sigma_{B}\right),
$$

we see that

$$
\widetilde{\Omega}(p, q)=\sup \left\{\frac{\tilde{f}^{*}\left(d \sigma_{B}\right)}{d \sigma_{X}}(p): \widetilde{f} \in \mathscr{B}(X), f(p)=0\right\},
$$

where $\mathscr{B}(X)$ is the set of all analytic functions $\widetilde{f}: X \rightarrow \boldsymbol{B}$. This shwos that $\widetilde{\Omega}(p, q)$ is actually independent of $q$ and $Y$. Relative to a fixed coordinate system at $p$ we have

$$
\frac{\tilde{f}^{*}\left(d \sigma_{\boldsymbol{B}}\right)}{d \sigma_{X}}(p)=\frac{\left|f^{\prime}(p)\right|}{\lambda_{X}(p)},
$$

if $f:(X, p) \rightarrow(\boldsymbol{B}, 0)$. A function $\tilde{f}$ in $\mathscr{B}(X)$ which maximizes $\left|\tilde{f}^{\prime}(p)\right|$ is called an Ahlfors function ([1], [2]) and $c_{B}(p)=\max \left\{\left|\widetilde{f}^{\prime}(p)\right|: \widetilde{f} \in\right.$ $\mathscr{B}(X)\}$ is called the analytic capacity metric. Therefore, $\widetilde{\Omega}(p, q)=$ $c_{B}(p) / \lambda_{X}(p)$.

For an annulus $\widetilde{\Omega}=\Omega$ and the quantity $\widetilde{\Omega}$ was investigated in [16]. Next, we show that generally $\Omega(p, q)$ is not uniformly less than one for a fixed $q$ as $p$ varies over $X$.

Proposition 4. Let $X$ be a bounded plane region. Suppose a point $\zeta \in \partial X$ has the property that two closed balls have $\zeta$ on their boundary, the interior of one ball lies entirely in $X$ and the interior of the other ball is in the exterior of $\Omega$. Then $\lim _{\sup _{p \rightarrow \xi}} \Omega(p, q)=1$ for any Riemann surface $Y$.

Proof. Let $I$ be the interior of the ball which is contained in $X$ and let $E$ be the exterior of the ball outside of $X$. Then $I \subset X \subset E$ and

$$
\lim _{p \rightarrow:} \frac{\lambda_{E}(p)}{\lambda_{J}(p)}=1
$$

when $p \rightarrow \zeta$ so that $p-\zeta$ makes with the interior normal at $\zeta$ an angle in absolute value less than $\alpha$, where $\alpha<\pi / 2$ [5, pp. 37-38]. Since $I \subset X \subset E$ we have

$$
\lambda_{I}(p) \geqq \lambda_{X}(p) \geqq \lambda_{E}(p)
$$

and

$$
\lambda_{I}(p)=c_{B}(p, I) \geqq c_{B}(p, X) \geqq c_{B}(p, E)=\lambda_{E}(p),
$$

so that 


$$
\frac{\lambda_{E}(p)}{\lambda_{I}(p)} \leqq \frac{c_{B}(p, X)}{\lambda_{X}(p)} \leqq \Omega(p, q) \leqq 1
$$

This establishes the result.

On the other hand, it is sometimes possible to bound $\Omega(p, q)$ above, independent of $q$.

THEOREM 7. Let $X$ be a Riemann surface which is not conformally equivalent to $\boldsymbol{B}$ or $\boldsymbol{B} \backslash\{0\}$. Suppose $Y$ is a Riemann surface with discrete modular spectrum and no point cycles. Given $p \in X$ there is a constant $\Omega(p)<1$ such that $\Omega(p, q) \leqq \Omega(p)$ for all $q \in Y$.

Proof. Let $\gamma$ be a closed curve based at $p$ with $M_{X}(\gamma)>0$. Given any $f \in N(X, Y)$ we have $0 \leqq M_{Y}(f \circ \gamma)<M_{X}(\gamma)$ and $M_{Y}(f \circ \gamma)=0$ if and only if $f \circ \gamma$ is freely homotopic to a point since $Y$ has no point cycles. Because $Y$ has discrete modular spectrum there are just finitely many numbers in the spectrum of $Y$ less than $M$, say $M_{1}, \cdots, M_{n}$ are these values. Set $M_{0}=0$. Let $N_{i}=\{f \in N(X, Y)$ : $\left.M_{Y}(f \circ \gamma)=M_{i}\right\}, 0 \leqq i \leqq n$, and define

$$
\Omega_{i}=\sup \left\{\frac{f^{*}\left(d \sigma_{Y}\right)}{d \sigma_{X}}(p): f \in N_{i}(X, Y)\right\} .
$$

It suffices to prove $\Omega_{i}<1,0 \leqq i \leqq n$.

First, we consider $1 \leqq i \leqq n$. Let $\pi:\left(A_{R}, \widetilde{p}\right) \rightarrow(X, p)$ be the annular covering surface determined by the free homotopy class $\{\gamma\}$. If $f \in N_{i}$, then let $\omega_{f}:\left(A_{s}, \widetilde{q}\right) \rightarrow(Y, f(p))$ be the annular covering surface defined by $\{f \circ \gamma\}$. Note that $S>R$ and $S$ is independent of $f$. Then $f$ induces a unique analytic function $\widetilde{f}:\left(A_{R}, \widetilde{p}\right) \rightarrow\left(A_{s}, \widetilde{q}\right)$ such that $\tilde{f}_{*}: \pi_{1}\left(A_{R}, \widetilde{p}\right) \rightarrow\left(A_{S}, \widetilde{q}\right)$ is an isomorphism. Thus, each $f \in N_{i}$ lifts to an analytic function $\tilde{f}: A_{R} \rightarrow A_{S}$ which satisfies $f \circ \pi=$ $\omega_{f} \circ \widetilde{f}$ and induces an isomorphism of fundamental groups; suppose $\tilde{N}_{i}$ is the set of all such lifts. Because coverings preserve the hyperbolic metric we have

$$
\Omega_{i}=\sup \left\{\frac{\widetilde{f}^{*}\left(d \sigma_{S}\right)}{d \sigma_{R}}(\widetilde{p}): \widetilde{f} \in \widetilde{N}_{i}\right\} .
$$

Note that $\tilde{N}_{i}$ is a normal family whose closure is compact. Therefore, if $\Omega_{i}=1$, then there is an analytic function $\widetilde{f}: A_{R} \rightarrow A_{S}$ in the closure of $\tilde{N}_{i}$ for which $\left(\tilde{f}^{*}\left(d \sigma_{s}\right) / d \sigma_{n}\right)(p)=1$. Then $\widetilde{f}$ must be a covering. But this covering can have only one sheet since $\tilde{f}_{*}$ is an isomorphism of $\pi_{1}\left(A_{R}, \widetilde{p}\right)$ onto $\pi_{1}\left(A_{S}, \widetilde{f}(\widetilde{p})\right)$. Hence, $\widetilde{f}$ is a conformal mapping of $A_{R}$ onto $A_{S}$ which is impossible since $R<S$. This contradiction shows that $\Omega_{i}<1,1 \leqq i \leqq n$.

Finally, we consider $i=0$. Suppose $f \in N_{0}$. Let $\omega:(\boldsymbol{B}, 0) \rightarrow$ 
$(Y, f(p))$ be a universal covering projection. Then $f$ lifts to an analytic function $\tilde{f}:\left(A_{R}, \widetilde{p}\right) \rightarrow(B, 0)$ which satisfies $f \circ \pi=\omega \circ \widetilde{f}$. As before let $\tilde{N}_{0}$ be the set of all such lifts. Then $\left|\tilde{f}^{\prime}(p)\right| \leqq c_{B}(\widetilde{p})$, where $c_{B}(z)|d z|$ is the analytic capacity metric for $A_{I i}$. Thus,

$$
\frac{\tilde{f}^{*}\left(d \sigma_{B}\right)}{d \sigma_{R}}(p) \leqq \frac{c_{B}(\widetilde{p})}{\lambda_{R}(\widetilde{p})}<1,
$$

where $d \sigma_{R}=\lambda_{R}(z)|d z|$ is the hyperbolic metric on $A_{R}$. This proves that $\Omega_{0} \leqq c_{B}(\widetilde{p}) / \lambda_{R}(\widetilde{p})$.

Added in proof. Recently, the question of $\mathrm{H}$. Hopf that was mentioned in Section 5 has been resolved. T. Jørgenson, A. Marden and C. Pommerenke (Two examples of covering surfaces, preprint) presented examples of Riemann surfaces that admit nontrivial selfcoverings.

\section{REFERENCES}

1. L. V. Ahlfors, Bounded analytic functions, Duke Math. J., 14 (1947), 1-11.

2. - Open Riemann surfaces and extremal problems on compact subregions, Comment. Math. Helv., 24 (1950), 100-134.

3. L. V. Ahlfors and L. Sario, Riemann surfaces, Princeton Math. Series, no. 26, Princeton Univ. Press, Princeton, N.J., 1960.

4. G. Aumann and C. Carathéodory, Ein Satz über die konforme Abbildung mehrfach zusammenhängender Gebiete, Math. Ann., 109 (1934), 756-763.

5. S. Bergman, The kernel function and conformal mapping, Math. Surveys no. 5, Amer. Math. Soc., Providence, R.I., 1970.

6. M. Heins, Extremal problems for functions analytic and single-valued in a doubly connected region, Amer. J. Math., 62 (1940), 91-106.

7. - A generalization of the Aumann-Carathéodory "Starrheitssatz," Duke Math. J., 8 (1941), 312-316.

8. — On a problem of Heinz Hopf, J. Math. Pure Appl., 37 (1958), 153-160.

9. M. Hervé, Quelques proprieties des transformations intérieurs d'un domain borné, Ann. Sci. Ecole Norm. Sup. Ser., 368 (1951), 125-184.

10. H. Huber, Über analytische Abbildungen Riemannscher Flächen in sich, Comment. Math. Helv., 27 (1953), 1-73.

11. J. A. Jenkins and N. Suita, On analytic self-mappings of Riemann surfaces, Math. Ann., 202 (1973), 37-56.

12. Y. Komatu and A. Mori, Conformal rigidity of Riemann surfaces, J. Math. Soc. Japan, 4 (1952), 302-309.

13. J. Lehner, Discontinuous groups and automorphic functions, Math. Surveys no. 8, Amer. Math. Soc., Providence, R.I., 1964.

14. A. Marden, I. Richards and B. Rodin, Analytic self-mappings of Riemann surfaces, J. Analyse Math., 18 (1967), 197-225.

15. W.S. Massey, Algebraic Topology: An Introduction, Harcourt, Brace and World, New York, 1967.

16. C. D. Minda, The Aumann-Carathéodory rigidity constant for doubly connected regions, (to appear in Ködai Math. J.).

Received December 5, 1978. Research supported in part by National Science Foundation Grant MCS78-02662. 


\section{PACIFIC JOURNAL OF MATHEMATICS}

\section{EDITORS}

DONALD BABBITT (Managing Editor)

University of California

Los Angeles, California 90024

Hugo RossI

University of Utah

Salt Lake City, UT 84112

C. C. MOORE and ANDrew OGG

University of California

Berkeley, CA 94720
J. DUgundjI

Department of Mathematics University of Southern California Los Angeles, California 90007

R. FinN aNd J. Milgram Stanford University Stanford, California 94305

\section{ASSOCIATE EDITORS}

E. F. BECKENBACH

B. H. NeUMANN

F. WOLF

K. YosHIDA

\section{SUPPORTING INSTITUTIONS}

UNIVERSITY OF BRITISH COLUMBIA CALIFORNIA INSTITUTE OF TECHNOLOGY UNIVERSITY OF CALIFORNIA MONTANA STATE UNIVERSITY UNIVERSITY OF NEVADA, RENO NEW MEXICO STATE UNIVERSITY OREGON STATE UNIVERSITY UNIVERSITY OF OREGON
UNIVERSITY OF SOUTHERN CALIFORNIA STANFORD UNIVERSITY UNIVERSITY OF HAWAII UNIVERSITY OF TOKYO UNIVERSITY OF UTAH WASHINGTON STATE UNIVERSITY UNIVERSITY OF WASHINGTON 


\section{Pacific Journal of Mathematics}

\section{Vol. 84 , No. 1 \\ May, 1979}

Michael James Beeson, Goodman's theorem and beyond ...............

Robert S. Cahn and Michael E. Taylor, Asymptotic behavior of multiplicities

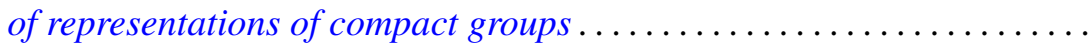

Douglas Michael Campbell and Vikramaditya Singh, Valence properties of

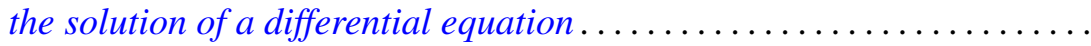

J.-F. Colombeau, Reinhold Meise and Bernard Perrot, A density result in spaces of Silva holomorphic mappings .....................

Marcel Erné, On the relativization of chain topologies .................

Le Baron O. Ferguson, Uniform and $L_{p}$ approximation for generalized

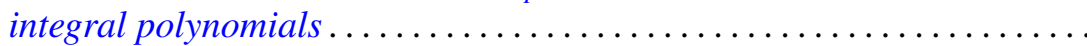

Kenneth R. Goodearl and David E. Handelman, Homogenization of regular rings of bounded index..................................

Friedrich Haslinger, A dual relationship between generalized

Abel-Gončarov bases and certain Pincherle bases .................

Miriam Hausman, Generalization of a theorem of Landau. .

Makoto Hayashi, 2-factorization in finite groups

Robert Marcus, Stochastic diffusion on an unbounded domain ........... 143

Isabel Dotti de Miatello, Extension of actions on Stiefel manifolds....

C. David (Carl) Minda, The hyperbolic metric and coverings of Riemann surfaces...

Somashekhar Amrith Naimpally and Mohan Lal Tikoo, On $T_{1}$-compactifications.

Chia-Ven Pao, Asymptotic stability and nonexistence of global solution for a semilinear parabolic equation ....

Shigeo Segawa, Harmonic majoration of quasibounded type ...

Sze-Kai Tsui and Steve Wright, The splitting of operator algebras ...

Bruce Williams, Hopf invariants, localization and embeddings of Poincaré complexes....

Leslie Wilson, Nonopenness of the set of Thom-Boardman maps ...

Alicia B. Winslow, There are $2^{\mathrm{c}}$ nonhomeomorphic continua in $\beta R^{n}-R^{n}$ 